\section{Excretion of Nitrogen by Leguminous Plants}

Writh reference to Prof. P. W. Wilson's communication upon this subject ${ }^{1} \mathrm{I}$ wish to make the following comments.

In our laboratory, where the excretion of nitrogen compounds from the leguminous root nodules has been for the first time definitely proved with a sterile culture system, hundreds of experiments have been carried out during the last ten years. In all experiments-both in sterile and in ordinary pot cultures - distinct excretion has practically always been detected, when suitable bacterial strains have been used for inoculation of red clover, white clover and pea, and the experimental conditions have been natural. Our experiments have not been restricted only to sand cultures, but similar experiments have also been carried out in clay and sandy loam soils, in kaolin medium, etc. The excretion of nitrogen compounds from the root nodules is therefore no incidental phenomenon which would appear only under certain artificial laboratory conditions. On the contrary, it occurs in experiments carried out under most natural conditions.

When Prof. Wilson could find no excretion in his numerous experiments during three years and now at last finds the phenomenon occurring in certain experiments, in others not, his experimental conditions must in some way differ from the natural ones. So far as is known to me, Prof. Wilson is using very coarse quartz sand as the substrate and the pot cultures are watered many times a day. According to our investigations the excretion depends greatly on the absorptive capacity of the medium. For example, with water cultures as well as with those containing glass beads, we have as a rule not been able to show any distinct excretion. Nor can any noticeable excretion be expected if the quartz sand particles are big.

From cultures grown on such media no conclusions can be drawn with regard to practical agriculture. Our experiments have been carried out partly in very fine quartz sand, which has a great absorptive capacity, partly in different soils. On the basis of these experiments, and particularly of those carried out in soil, it can be definitely concluded that the excretion is a phenomenon which must occur in the field and plays an important part in practical agriculture. The benefit to non-legumes in associated cultures with legumes, a fact which has been known in practical agriculture for thousands of years, has been explained through the excretion phenomenon. I refer to our publication concerning the associated cultures appearing in the October issue of the Journal of Agricultural Science and also to my paper presented before the Fourth Agricultural Grassland Congress at Aberystwyth in July this year.

The extent of excretion varies considerably even in parallel experiments and depends, as $I$ have often emphasized, on many factors, of which so far only a part is known to us. Such factors are, for example, bacterial strain, amount of nodules, host plant, medium and nitrate content of the medium ${ }^{2}$.

At least with the plants employed by us, the excretion can always be shown, and with our recent knowledge of the factors effecting the excretion. In associated cultures of lucerne and rye-grass, Thornton and Nicol (1934), in Rothamsted have detected distinct excretion. If some species of legumes (for example, soy) should differ in this respect, it certainly does not mean that the excretion phenomenon has no importance in agriculture. However, I consider that, even with soy, thorough investigations must first be carried out using different bacterial strains in inoculating different species of soy and natural media, before anything definite can be stated.

\section{Artturi I. Virtanten.}

Biochemical Institute, Helsinki.

Aug. 31.

1 NATURE, 140, 154 (1937).

'See J. Agr. Sci., July 1937.

A PREvious communication ${ }^{1}$ referred to experiments with inoculated soybeans (variety "Manchu") growing under normal conditions in sand culture, in which I was unable to detect any excretion of nitrogenous substances from the nodules into the rooting medium, as demonstrated by Virtanen ${ }^{2}$ and his collaborators in other legumes, especially the pea. Further experiments, in which the sand from the culture pots was submitted to direct Kjeldahl analysis without previous drying, have also yielded negative results. The maximum difference between nitrogen contents of the sand from inoculated and control pots was only $2.5 \mathrm{mgm}$., after four months' growth, during which time $350 \mathrm{mgm}$. of nitrogen was fixed within the nodules of the five plants of the inoculated pot. Barley plants grown in the same pots as nodulated soybeans derived no benefit from the association, confirming the absence of excretion. Three different strains of bacillus have been tried, including two of the most efficient in fixation from the Wisconsin collection, all with negative results. It is therefore clear that certain measurements of fixation and transfer of nitrogen made in a previous investigation $^{3}$ under similar growth conditions are complete in themselves and do not require amendment.

Negative results have also been obtained with broad bean (Vicia Faba L.) variety "Longpod", both from sand analyses and from examination of test plants grown with the nodulated beans. The fixation here amounted to $275 \mathrm{mgm}$. for two plants.

It is impossible to say at present whether these negative results arise from the absence of excretion from these particular legumes under any conditions, or from the lack in the present experiments of certain undefined conditions necessary for excretion to proceed. Ludwig and Allison ${ }^{4}$ and Wilson ${ }^{5}$ have also been unable to find excretion in a number of legumes. The plant cultures of the present experiments were not strictly sterile in the sense that some of Virtanen's were, but since the latter author finds excretion in both sterile and ordinary open cultures, it is clear that absence of complete sterility is not a reason for negative results. It is possible that differences in anatomical details of the outer nodule tissues may account for any variations in excretion between different legumes that may finally be established.

With the pea (Pisum sativum L.) variety "Gradus", we have obtained a maximum excretion of $7 \mathrm{mgm}$. nitrogen per pot of three plants, the fixation being 60 mgm. (bacillus strain Virtanen HX). Barley 